\title{
Presaddle and postsaddle dissipative effects in fission using complete kinematics measurements
}

J. L. Rodríguez-Sánchez, ${ }^{1}$ J. Benlliure,,${ }^{1}$ J. Taieb,${ }^{2}$ H. Alvarez-Pol, ${ }^{1}$ L. Audouin, ${ }^{3}$ Y. Ayyad,,${ }^{1, *}$ G. Bélier, ${ }^{2}$ G. Boutoux,${ }^{2, \dagger}$ E. Casarejos, ${ }^{4}$ A. Chatillon, ${ }^{2}$ D. Cortina-Gil, ${ }^{1}$ T. Gorbinet, ${ }^{2}$ A. Heinz,${ }^{5}$ A. Kelić-Heil,${ }^{6}$ B. Laurent, ${ }^{2}$ J.-F. Martin, ${ }^{2}$ C. Paradela,,${ }^{1,}$ E. Pellereau, ${ }^{2}$ B. Pietras, ${ }^{1}$ D. Ramos, ${ }^{1}$ C. Rodríguez-Tajes, ${ }^{1,7}$ D. M. Rossi, ${ }^{6}$ H. Simon, ${ }^{6}$ J. Vargas, ${ }^{1}$ and B. Voss ${ }^{6}$

${ }^{1}$ Universidad de Santiago de Compostela, E-15782 Santiago de Compostela, Spain

${ }^{2}$ CEA, DAM, DIF, F-91297 Arpajon, France

${ }^{3}$ Institut de Physique Nucléaire d'Orsay, F-91406 Orsay, France

${ }^{4}$ Universidad de Vigo, E-36200 Vigo, Spain

${ }^{5}$ Chalmers University of Technology, SE-412 96 Gothenburg, Sweden

${ }^{6}$ GSI-Helmholtzzentrum für Schwerionenforschung GmbH, D-64291 Darmstadt, Germany

${ }^{7}$ Grand Accélérateur National d'Ions Lourds, F-14076 Caen Cedex 05, France

(Received 12 February 2016; published 2 December 2016)

\begin{abstract}
A complete kinematics measurement of the two fission fragments was used for the first time to investigate fission dynamics at small and large deformations. Fissioning systems with high excitation energies, compact shapes, and low angular momenta were produced in inverse kinematics by using spallation reactions of lead projectiles. A new generation experimental setup allowed for the first full and unambiguous identification in mass and atomic number of both fission fragments. This measurement permitted us to accurately determine fission cross sections, the charge distribution, and the neutron excess of the fission fragments as a function of the atomic number of the fissioning system. These data are compared with different model calculations to extract information on the value of the dissipation parameter at small and large deformations. The present results do not show any sizable dependence of the nuclear dissipation parameter on temperature or deformation.
\end{abstract}

DOI: 10.1103/PhysRevC.94.061601

Fission is the clearest example of large-scale collective excitations in nuclei. Since its discovery by Hahn et al. in 1939 [1,2], progress in the understanding of the fission process has been driven by new experimental results. Although the first explanation of nuclear fission was provided by Bohr and Wheeler [3] using the transition-state model, new experimental observations revealed the complexity of nuclear fission and provided new challenges for theory. In particular, the investigation of pre- and postscission neutron multiplicities [4,5], giant dipole resonance (GDR) $\gamma$-ray emission $[6,7]$, multiplicities of charged particles [8,9], and fission and evaporation cross sections [10-12] established that the dynamical evolution of the fissioning system cannot be explained just in terms of the statistical model of Bohr and Wheeler [3]. These results suggested that the understanding of the fission process requires a dynamical approach, describing the coupling of intrinsic and collective excitations of the nuclear constituents. Here, the transfer of energy between the intrinsic and collective excitations is governed by dissipation due to fluctuating forces. In this context, transport models based on the Fokker-Planck [13] or the Langevin equations [14] have proven to be suitable tools for the description of the collective evolution of nuclei.

However, the dynamics of fission is still far from being fully understood because our theoretical and experimental knowledge is not yet complete. Several works claim that the reduced dissipation parameter $(\beta)$, defined according to Ref. [13],

\footnotetext{
*Present address: National Superconducting Cyclotron Laboratory, Michigan State University, East Lansing, MI 48824-1321, USA.

†Present address: Université Bordeaux, F-33405 Talence, France.

${ }^{\ddagger}$ Present address: EC-JRC, Institute for Reference Materials and Measurements, Retieseweg 111, B-2440 Geel, Belgium.
}

could change with the nuclear deformation or with the nuclear temperature $[4,7,15]$. These ideas are still under debate because they could be biased by experimental conditions [16].

The ground-to-saddle dynamics and more particularly the presence of transient time effects, which corresponds to the time required to reach $90 \%$ of the stationary fission flux across the barrier, have been actively debated during the past decade [8,10,17-20]. Experiments taking advantage of spallation- and fragmentation-induced fission reactionsshed some light on the problem [21,22]. The combined use of the inverse kinematics technique and an efficient detection setup made it possible to identify fission reactions and determine the atomic number of the final fission fragments very accurately. The sum of the charges of both fission fragments was used as a measurement of the initial excitation energy while partial fission cross sections and the width of the charge distribution of the fission fragments were shown to be sensitive to presaddle dynamical effects $[18,20,23,24]$. The measurements obtained for many different fissioning systems over a broad range in fissility and temperature were compatible with a constant value of the reduced dissipation parameter at small deformations of $\beta=4.5 \times 10^{21} \mathrm{~s}^{-1}$, corresponding to transient times $\left(\tau_{\text {trans }}\right)$ between 1.0 and $3.3 \times 10^{-21} \mathrm{~s}$. These results are compatible with the ones obtained from the investigation of some fusion reactions [16,25]. Moreover, the overall good description of the data over a broad range of excitation energies also validated previous conclusions on the temperature independence of the dissipation parameter [11,12] and references therein.

Postsaddle dissipative effects have been mostly investigated by measuring prescission particles and $\gamma$ rays emitted in fusion-fission reactions [15,26-28]. In some cases, fission or evaporation-residue cross sections were also measured, constraining presaddle effects. Because of the 


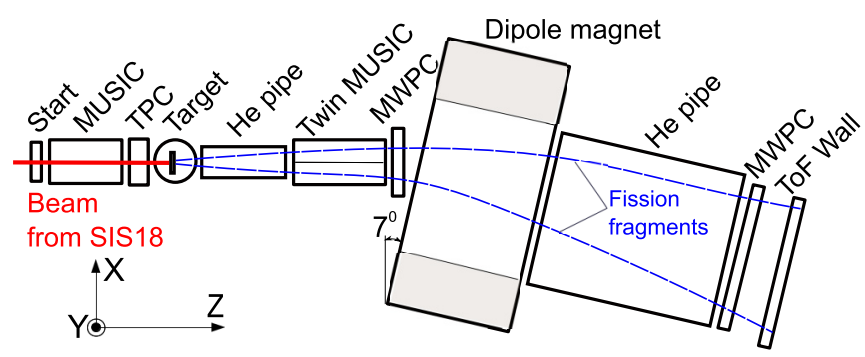

FIG. 1. Top schematic view of the experimental setup. Sizes are not to scale.

different saddle-to-scission deformations lengths reached in these reactions, pre- and postsaddle dissipative effects were enhanced by investigating low- and high-fissility systems, respectively. Most of the results obtained in these works seem compatible with presaddle reduced dissipation parameters between 2 and $6 \times 10^{21} \mathrm{~s}^{-1}$, while the saddle-to-scission dynamics is better described by using larger values around $30 \times$ $10^{21} \mathrm{~s}^{-1}$. However, these conclusions contradict recent results obtained with sophisticated Langevin calculations [25,29], where the value of the dissipation parameter slightly decreases with deformation.

To go further, we propose the use of complete kinematics measurements of the two fission fragments to study the deformation dependence of the dissipation parameter. For this purpose we introduce the neutron excess of the final fission fragments as a new experimental observable sensitive to dissipative effects at large deformations. This observable, together with others previously investigated [30], is used to deduce the value of the dissipation parameter before and after the saddle point.

As discussed in the pioneering work of Grangé and collaborators [31], the optimal conditions for investigating dissipative and transient effects in fission are the use of spherical fissioning systems with low angular momentum and high excitation energies $\left(E^{*}>100 \mathrm{MeV}\right)$. These conditions also represent a good approach to study dissipative effects at large deformations because high excitation energies also favor the evaporation of particles beyond the saddle-point configuration [15]. To reproduce this ideal scenario, we use proton-induced fission reactions on ${ }^{208} \mathrm{~Pb}$ at relativistic energies. Despite the shorter saddle-to-scission path length of the fissioning nuclei produced in this reaction, the sensitivity to postsaddle dynamical effects is expected because higher energies increase the emission of pre- and postsaddle particles, facilitating the investigation of ground-to-saddle and saddleto-scission dissipative effects.

The experiment was performed at the GSI facilities in Darmstadt (Germany), where the SIS18 synchrotron was used to accelerate ions of ${ }^{208} \mathrm{~Pb}$ at $500 \mathrm{~A} \mathrm{MeV}$ with an intensity around $10^{5}$ ions/s. The primary beam was then guided to the experimental setup where fission reactions were induced in a cylindrical target filled with liquid hydrogen ( $\left.\sim 85 \mathrm{mg} / \mathrm{cm}^{2}\right)$ [32].

A schematic drawing of the experimental setup is shown in Fig. 1. The use of the inverse kinematics allowed us to separate fission from other reaction channels and also facilitated the identification of both fission fragments. Fission events were identified by using energy-loss measurements performed with a double multisampling ionization chamber (Twin MUSIC), providing the atomic number of the two fission fragments with a resolution better than 0.43 charge units full width at half maximum (FWHM) [24]. In addition, the tracking capabilities of this detector permitted us to select fission events produced at the target position [32]. Mass numbers were determined from magnetic rigidity and time-of-flight measurements with an average resolution of $\triangle A / A \sim 0.63 \%$ (FWHM) [24], providing for the first time a complete and unambiguous identification in mass and atomic number of both fission fragments. Other details can be found in Ref. [24].

For the interpretation of the observables, we compare the data to model calculations. These calculations were done by using the code INCL4.6 [33] to describe the first stage of the reaction induced by energetic protons according to the intranuclear cascade approach. The de-excitation of the resulting remnants was described by using the code ABLA07 [34]. The fission decay width is described by using an analytical approximation of the solution of the Fokker-Planck equation describing fission as a diffusion process across the fission barrier [17]. Fission barriers are calculated according to the finite-range liquid-drop model of Sierk [35], considering shell effects [36]. The nuclear level densities are obtained by using the back-shifted Fermi-gas formulation [37,38], together with Ignatyuk's parametrization [38] for the level density parameter. Finally, the mass and atomic-number distributions of the fission fragments are described by the semiempirical model proposed in Ref. [39]. In addition, ABLA07 takes into account the emission of $\gamma$ rays, neutrons, light-charged particles, and intermediate-mass fragments (IMFs), according to the Weisskopf formalism [40].

To include the fission dynamics beyond the saddle point, we have used the pioneering model proposed by Hofmann and Nix [41], who found an analytical solution of the average saddle-to-scission time based on the dynamical picture of Kramers [42], taking into account the deformations of the compound systems at the saddle configuration [43] and the deformations of the fission fragments at the scission point [36]. This dynamical time can be compared with the statistical evaporation time obtained from the sum of the particle decay widths to determine the number of particles emitted between the saddle and scission points. If the saddle-to-scission time of the fissioning system is longer than the statistical evaporation time the fissioning system could emit additional particles, mainly neutrons and $\gamma$ rays, during that phase, increasing the prescission particle multiplicities. Similar model calculations were used in many of the previous works claiming a deformation-dependent dissipation parameter [4,26,27].

Following Refs. [18,20], we have used the partial fission cross sections obtained in this experiment to investigate dissipative effects at small deformations. This observable is displayed in Fig. 2 (solid circles) as a function of the sum of the atomic numbers of the two fission fragments $\left(Z_{1}+Z_{2}\right)$, which corresponds in good approximation to the atomic number of the fissioning system. The values of $\left(Z_{1}+Z_{2}\right)$ are correlated with the violence of the reaction or excitation energy of the remnants [18]. In our case, they cover a large 


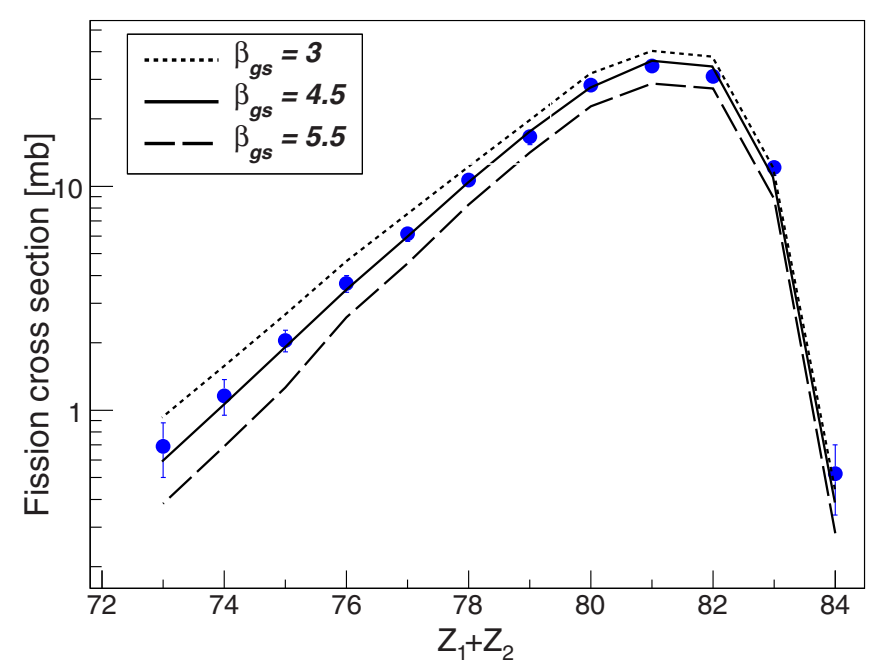

FIG. 2. Fission cross sections as a function of the atomic number of the fissioning nuclei. The lines represent INCL4.6+ABLA07 calculations for different values of the reduced dissipation parameter $\beta_{g s}$ in units of $10^{21} \mathrm{~s}^{-1}$.

range in excitation energies (up to $350 \mathrm{MeV}$ ), providing us with the possibility to investigate the temperature dependence of the dissipation parameter. In the figure, we compare this observable with model calculations for different values of the reduced dissipation parameter $\beta$. We conclude that a constant value of this dissipation parameter from ground to saddle $\left(\beta_{g s}\right)$ of $(4.5 \pm 0.5) \times 10^{21} \mathrm{~s}^{-1}$ (solid line) provides the best description of these data. The description of the data over the complete range in $Z_{1}+Z_{2}$ by using a constant value of the dissipation parameter confirms the conclusions obtained in previous works with respect to the magnitude and temperature independence of this parameter at small deformations [18,20,22].

The model dependence of these conclusions, common to the interpretation of any experiment addressing the fission dynamics, has been constrained by using different reactions and observables. The description of the range of compound nuclei produced in spallation reactions, whose characteristics cannot be accessed experimentally, has been validated describing the isotopic composition of final residual nuclei in reactions with nonfissile nuclei [33]. Moreover, the ground-to-saddle dynamics was constrained by measuring the atomic numbers of both fission fragments $[16,18,23,30]$ or the isotopic composition of one of the two fragments $[24,44,45]$. We can then conclude that these calculations provide a reasonable description of the fissioning nuclei at the saddle-point deformation. Therefore, they can be considered as a suitable tool to investigate dissipative effects at large deformations by using additional observables sensitive to the saddle-to-scission dynamics.

For the investigation of the saddle-to-scission dynamics we studied first the prescission neutron multiplicities as a function of the atomic number of the fissioning system $\left(Z_{1}+Z_{2}\right)$, as shown in Fig. 3(a) (solid circles). This observable was obtained by using the neutron and atomic numbers of the two fission fragments, as well as their velocities. The atomic numbers $Z_{1}$ and $Z_{2}$ together with the velocities [24] were used
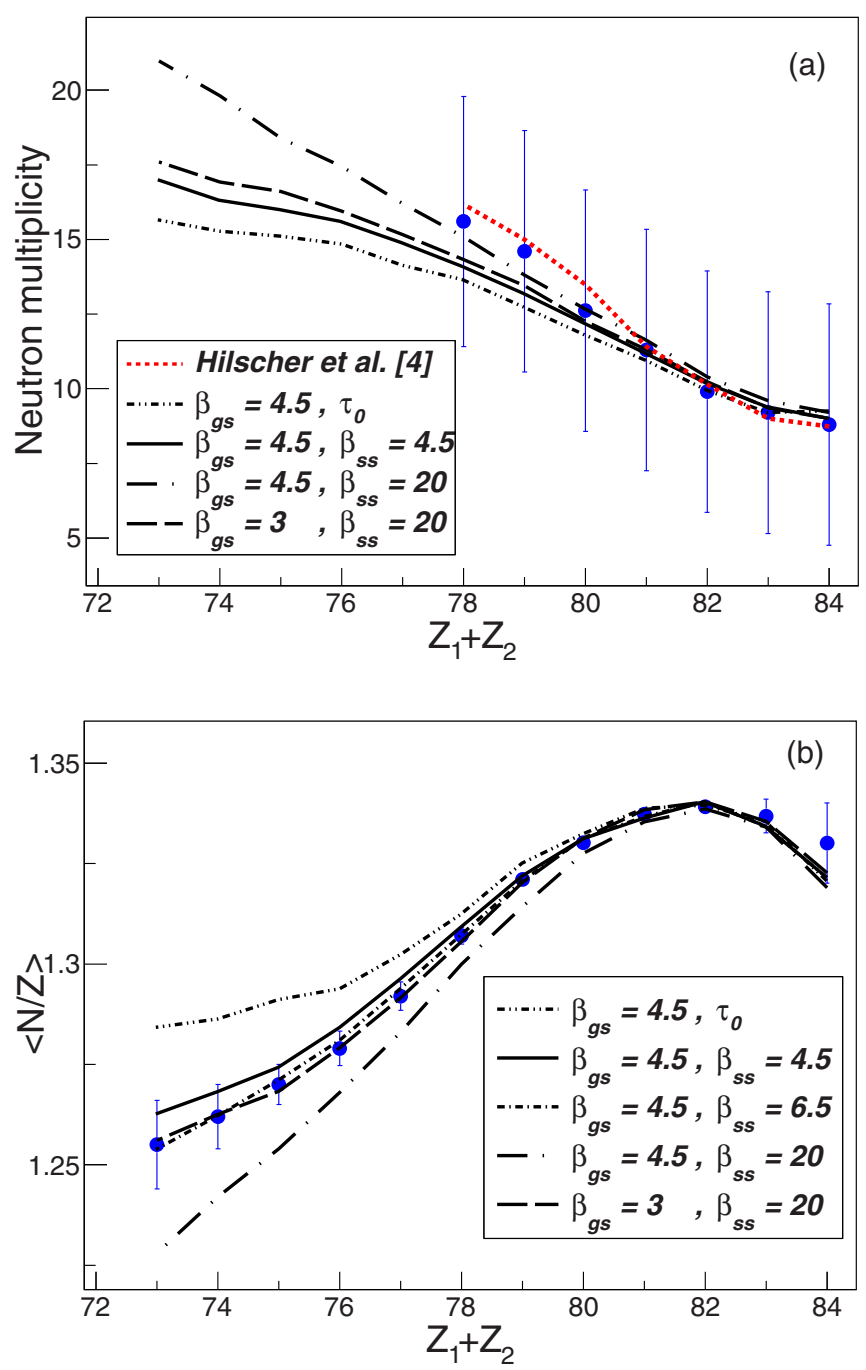

FIG. 3. Prescission neutron multiplicities (a) and average neutron excess of the final fission fragments (b) as a function of the atomic number of the fissioning nuclei. The lines represent model calculations for different values of the dissipation parameters in units of $10^{21} \mathrm{~s}^{-1}$.

to reconstruct the neutron number of the fissioning system at scission $\left(N_{\text {fiss }}^{s c i}\right)$ by using the Wilkins model [46] and by assuming momentum conservation at scission according to Ref. [47]. The difference between the neutron number of the projectile $\left(N_{\text {proj }}\right)$ and that of the fissioning system at scission provided us, event by event, the prescission neutron multiplicity $\left(v_{n}^{\text {presci }}=N_{\text {proj }}-N_{\text {fiss }}^{s c i}\right)$ obtained for fissioning systems with $Z_{1}+Z_{2}>77$, because other decay channels $(d, t, \mathrm{He}$, and IMFs) are not negligible for lighter fissioning systems with higher excitation energies. This multiplicity is a sum of the neutrons emitted during the cascade stage and the de-excitation of the compound nuclei. However, this fact does not represent a problem because the neutrons emitted in the cascade process represent at most $20 \%$ of the total prescission neutrons [33]. Moreover, these neutrons are not related with the de-excitation of the compound systems and thus are not sensitive to dissipation. Finally, the associated uncertainties 
are mainly attributed to the accuracy in the measurement of the velocities of the fission fragments in the reference frame of the incoming projectiles, used to reconstruct the fissioning system at scission.

Figure 3(a) shows the expected increase of the prescission neutron multiplicities with the excitation energy (lightest fissioning systems) [4]. As shown in Ref. [24], the average excitation energy increases from 80 up to $350 \mathrm{MeV}$ when decreasing the atomic number of the fissioning system. We also display the neutron-multiplicity systematics proposed by Hilsher and Rossner [4], which was found by investigating fusion-fission reactions of different nuclei at excitation energies from 6 up to $200 \mathrm{MeV}$. The good agreement with this systematics validates our neutron multiplicity.

In the same figure, the double-dot-dashed line represents a calculation assuming a ground-to-saddle transient time, corresponding to a value of $\beta_{g s}=4.5 \times 10^{21} \mathrm{~s}^{-1}$, and a saddleto-scission time $\left(\tau_{o}\right)$ obtained from the Kramer's stationary solution for $\beta s s=0$ according to Ref. [41]. On the other hand, the solid and dot-long-dashed lines represent similar calculations considering a reduced saddle-to-scission dissipation parameter $\left(\beta_{s s}\right)$ of $4.5 \times 10^{21} \mathrm{~s}^{-1}$ and $20 \times 10^{21} \mathrm{~s}^{-1}$, respectively. As can be seen, one can only expect a sensitivity to the different model calculations at high excitation energies, corresponding to small $Z_{1}+Z_{2}$ values. Even in that case, one would need a much better prescision in the neutron multiplicities to reach any unambiguous conclusion on the saddle-to-scission dynamical effects. However, the sensitivity of neutron and $\gamma$-ray prescission multiplicities to presaddle dynamics, which was widely exploited in the past, is much stronger.

The measurement of the neutron excess of the two final fission fragments $(\langle N / Z\rangle)$ represents an alternative to investigate dissipative effects at large deformations. Even if this observable depends on prescission and postscission neutron and proton evaporation, the fission time and the $\gamma$-ray emission may alter the relative probabilities for neutron and proton emission. At short fission times (small saddle-to-scission dissipation value) the system mostly emits neutrons up to the scission point, while for longer fission times (larger saddle-toscission dissipation value) $\gamma$-ray emission channel competes with neutrons and at even longer times proton emission becomes also relevant. All these considerations explain the sensitivity of the final $(\langle N / Z\rangle)$ of the fission fragments to dissipation. Furthermore, in our experiment the neutron excess of the final fission fragments is measured very accurately and with high efficiency, resulting in a better constraint for model calculations.

The average neutron excess of the final fission fragments is displayed as a function of the atomic number of the fissioning nuclei in Fig. 3(b). This observable covers a large range in fissioning systems from $Z_{1}+Z_{2}=73$ up to $Z_{1}+Z_{2}=84$ thanks to the high efficiency of the SOFIA setup. In the figure, we also show the results of our model calculations. As can be observed, a statistical calculation (double-dot-dashed line) reproduces the neutron excess for the heaviest fissioning systems, where the saddle-to-scission neutron evaporation is negligible due to the low excitation energies. However, this calculation clearly overestimates the neutron excess for the lightest fissioning systems. This overestimation indicates the need for a longer saddle-to-scission time, increasing the prescission neutron evaporation probability at high excitation energies. Calculations assuming $\beta_{g_{s}}=4.5 \times 10^{21} \mathrm{~s}^{-1}$ and different values for $\beta_{s s}: 4.5 \times 10^{21} \mathrm{~s}^{-1}$ (solid line), $6.5 \times$ $10^{21} \mathrm{~s}^{-1}$ (dot-short-dashed line), and $20 \times 10^{21} \mathrm{~s}^{-1}$ (dot-longdashed line) are also shown. In this case, the accuracy in the measurement of the neutron excess of the final fission fragments confirms the sensitivity of this observable to the saddle-to-scission dissipation parameter. This benchmark also allows us to establish that calculations considering a reduced dissipation parameter between $4.5 \times 10^{21} \mathrm{~s}^{-1}$ (solid line) and $6.5 \times 10^{21} \mathrm{~s}^{-1}$ (dot-short-dashed line) can describe the average neutron excess for the full range of fissioning systems.

In Figs. 3(a) and 3(b) we also show a calculation assuming $\beta_{g s}=3 \times 10^{21} \mathrm{~s}^{-1}$ and $\beta_{s s}=20 \times 10^{21} \mathrm{~s}^{-1}$ (long-dashed line) according to the results found in Refs. [15,26-28]. This calculation also provides a good description of the neutron excess of the fission fragments. The reason why two combinations of pre- and postsaddle dissipative parameters describe the data is that the observable we use, as well as the neutron multiplicities, is sensitive to the dynamics of the complete fission path until the scission point. Therefore, to investigate the saddle-to-scission dynamics in further detail with these observables one needs additional measurements providing an accurate description of the presaddle dynamics. This is clearly shown in Fig. 2 where the precise and accurate determination of the partial fission cross sections and the atomic number of the fission fragments allow us to rule out a ground-to-saddle dissipation parameter of $\beta_{g s}=3 \times 10^{21} \mathrm{~s}^{-1}$.

This result indicates that ground-to-saddle and ground-toscission observables can be described by using almost the same dissipation parameter at small and large deformations (around $4.5 \times 10^{21} \mathrm{~s}^{-1}$ ). This weak dependence of the dissipation parameter on deformation is in agreement with the predictions of the one-body chaos-weighted wall formula [48]. Moreover, the magnitude of the dissipation parameter at large deformation derived in this work is also in good agreement with the study of fusion reactions by using the linear response theory, taking the fluctuation-dissipation theorem and the width of the single-particle states into account [49].

The final validation of these conclusions could be brought by using advanced transport model calculations describing the measurements obtained in this work. Unfortunately, the variety of initial fissioning systems produced in spallation reactions prevent such calculations for the time being.

In our opinion, the previously obtained deformation dependence of the dissipation parameter [15,26-28,50] could be biased by the imprecise characterization of the ground-tosaddle dynamics. This fact could be caused by the difficulty in describing the initial configuration of the fissioning nuclei, namely angular momentum and deformation, inherent to fusion reactions.

In conclusion, the complete kinematics measurement of the fission fragments produced in spallation-induced fission reactions gives access to precise information on the fission dynamics. The low angular momentum and high excitation energies of the fissioning nuclei produced in these reactions provide optimal conditions for this investigation. The precise 
selection of fission events and the accurate identification in atomic and mass number of the fission fragments, based on the use of inverse kinematics and a state-of-the-art experimental device, made it possible to use several independent observables constraining nuclear dissipation at small and large deformations. The comparison of the partial fission cross sections with our model calculations permitted us to determine the magnitude of the dissipation parameter at small deformations, obtaining a value of $\beta_{g s}=(4.5 \pm 0.5) \times 10^{21} \mathrm{~s}^{-1}$.

The average neutron excess of the final fission fragments, displayed as a function of the atomic number of the fissioning nucleus, is used in this work for the first time to investigate dissipative effects specifically at large deformations. Model calculations have demonstrated that this innovative observable is very sensitive to dissipation provided that sufficiently high excitation energies, leading to a minimum saddle-to-scission neutron emission, are reached. This analysis yields a value for the reduced dissipation parameter at large deformations $\left(\beta_{s s}\right)$ between 4.5 and $6.5 \times 10^{21} \mathrm{~s}^{-1}$.

The similitude between the values of the dissipation parameter required to describe the fission dynamics before and after the saddle point excludes any strong dependence of this parameter with deformation. Moreover, the large range in excitation energy covered by the present data and their description by using a constant value of dissipation also exclude any strong dependence of this parameter with temperature.

The authors are grateful to the GSI accelerator staff for providing an intense and stable beam of ${ }^{208} \mathrm{~Pb}$. We also gratefully acknowledge J.-M. Gheller and S. Leray for providing the liquid-hydrogen target and N. Kurz and A. Prochazka for their technical support during the experiment. This work was partially supported by the European Commission under Projects No. ANDES-FP7-249671 and No. CHANDA-FP7605203, the Spanish Ministry of Economy and Competitiveness under Projects No. FPA2010-22174-C02, No. FPA201347831-C2-1-P, and No. Consolider-CPAN-CSD2007-00042, and the Regional Government of Galicia under the program Grupos de Referencia Competitiva 2013-011. One of us, C.R.T., also acknowledges the support of the Spanish Ministry of Education under a grant of postdoctoral mobility (2011) administered by FECYT.
[1] L. Meitner and O. R. Frisch, Nature (London) 143, 239 (1939).

[2] O. Hahn and F. Strassmann, Naturwissenschaften 27, 11 (1939).

[3] N. Bohr and J. A. Wheeler, Phys. Rev. 56, 426 (1939).

[4] D. Hilscher and H. Rossner, Ann. Phys. Fr. (Paris) 17, 471 (1992).

[5] D. J. Hinde, D. Hilscher, H. Rossner, B. Gebauer, M. Lehmann, and M. Wilpert, Phys. Rev. C 45, 1229 (1992).

[6] M. Thoennessen and G. F. Bertsch, Phys. Rev. Lett. 71, 4303 (1993).

[7] D. J. Hofman, B. B. Back, and P. Paul, Phys. Rev. C 51, 2597 (1995).

[8] J. P. Lestone, Phys. Rev. Lett. 70, 2245 (1993).

[9] J. P. Lestone et al., Nucl. Phys. A 559, 277 (1993).

[10] L. G. Moretto, K. X. Jing, R. Gatti, G. J. Wozniak, and R. P. Schmitt, Phys. Rev. Lett. 75, 4186 (1995).

[11] J. P. Lestone and S. G. McCalla, Phys. Rev. C 79, 044611 (2009).

[12] W. Ye and N. Wang, Phys. Rev. C 87, 014610 (2013).

[13] P. Grangé and H. A. Weidenmüller, Phys. Lett. B 96, 26 (1980).

[14] Y. Abe et al., Phys. Rep. 275, 49 (1996).

[15] P. Fröbrich and I. Gontchar, Nucl. Phys. A 556, 281 (1993).

[16] C. Schmitt, K.-H. Schmidt, A. Kelić, A. Heinz, B. Jurado, and P. N. Nadtochy, Phys. Rev. C 81, 064602 (2010).

[17] B. Jurado, K.-H. Schmidt, and J. Benlliure, Phys. Lett. B 553, 186 (2003).

[18] B. Jurado, C. Schmitt, K.-H. Schmidt, J. Benlliure, T. Enqvist, A. R. Junghans, A. Kelic, and F. Rejmund, Phys. Rev. Lett. 93, 072501 (2004).

[19] V. Tishchenko, C.-M. Herbach, D. Hilscher, U. Jahnke, J. Galin, F. Goldenbaum, A. Letourneau, and W.-U. Schroder, Phys. Rev. Lett. 95, 162701 (2005).

[20] C. Schmitt, P. N. Nadtochy, A. Heinz, B. Jurado, A. Kelić, and K.-H. Schmidt, Phys. Rev. Lett. 99, 042701 (2007).

[21] K.-H. Schmidt et al., Nucl. Phys. A 665, 221 (2000).

[22] Y. Ayyad, J. Benlliure, E. Casarejos, H. Álvarez-Pol, A. Bacquias, A. Boudard, M. Caamaño, T. Enqvist, V. Föhr, A. Kelić-Heil, K. Kezzar, S. Leray, D. Mancusi, C. Paradela,
D. Pérez-Loureiro, R. Pleskać, J. L. Rodríguez-Sánchez, and D. Tarrío, Phys. Rev. C 89, 054610 (2014).

[23] Y. Ayyad, J. Benlliure, J. L. Rodríguez-Sánchez, A. Bacquias, A. Boudard, E. Casarejos, T. Enqvist, M. Fernandez, V. Henzl, V. Henzlova, B. Jurado, A. Kelic-Heil, T. Kurtukian, S. Lukic, P. Nadtochy, D. Pérez-Loureiro, R. Pleskac, F. Farget, M. V. Ricciardi, K.-H. Schmidt, C. Schmitt, and S. N. Ngoc, Phys. Rev. C 91, 034601 (2015).

[24] J. L. Rodríguez-Sánchez et al., Phys. Rev. C 91, 064616 (2015)

[25] E. Vardaci, P. N. Nadtochy, A. Di Nitto, A. Brondi, G. La Rana, R. Moro, P. K. Rath, M. Ashaduzzaman, E. M. Kozulin, G. N. Knyazheva, I. M. Itkis, M. Cinausero, G. Prete, D. Fabris, G. Montagnoli, and N. Gelli, Phys. Rev. C 92, 034610 (2015).

[26] N. P. Shaw, I. Diószegi, I. Mazumdar, A. Buda, C. R. Morton, J. Velkovska, J. R. Beene, D. W. Stracener, R. L. Varner, M. Thoennessen, and P. Paul, Phys. Rev. C 61, 044612 (2000).

[27] I. Diószegi, N. P. Shaw, A. Bracco, F. Camera, S. Tettoni, M. Mattiuzzi, and P. Paul, Phys. Rev. C 63, 014611 (2000).

[28] I. Diószegi, N. P. Shaw, I. Mazumdar, A. Hatzikoutelis, and P. Paul, Phys. Rev. C 61, 024613 (2000).

[29] P. N. Nadtochy, E. G. Ryabov, A. E. Gegechkori, Yu. A. Anischenko, and G. D. Adeev, Phys. Rev. C 89, 014616 (2014).

[30] J. L. Rodríguez-Sánchez et al., Phys. Rev. C 92, 044612 (2015).

[31] P. Grangé, J.-Q. Li, and H. A. Weidenmüller, Phys. Rev. C 27, 2063 (1983).

[32] J. L. Rodríguez-Sánchez et al., Phys. Rev. C 90, 064606 (2014).

[33] A. Boudard, J. Cugnon, J.-C. David, S. Leray, and D. Mancusi, Phys. Rev. C 87, 014606 (2013).

[34] A. Kelić, M. V. Ricciardi, and K.-H. Schmidt, Proceedings of Joint ICTP-IAEA Advanced Workshop on Model Codes for Spallation Reactions, ICTP Trieste, Italy, 4-8 February 2008, edited by D. Filges, S. Leray, Y. Yariv, A. Mengoni, A. Stanculescu, and G. Mank (IAEA INDC (NDS) - 530, Vienna, 2008), pp. 181-221. 
[35] A. J. Sierk, Phys. Rev. C 33, 2039 (1986).

[36] P. Möller, J. R. Nix, W. D. Myers, and W. J. Swiatecki, At. Data Nucl. Data Tables 59, 185 (1995).

[37] H. A. Bethe, Phys. Rev. 50, 332 (1936).

[38] A. V. Ignatyuk, M. G. Itkis, V. N. Okolovich, G. N. Smirenkin, and A. S. Tishin, Yad. Fiz. 21, 1185 (1975) [Sov. J. Nucl. Phys. 21, 612 (1975)].

[39] J. Benlliure et al., Nucl. Phys. A 628, 458 (1998).

[40] V. F. Weisskopf and D. H. Ewing, Phys. Rev. 57, 472 (1940).

[41] H. Hofmann and J. R. Nix, Phys. Lett. B 122, 117 (1983).

[42] H. A. Kramers, Physica (Amsterdam, Neth.) 7, 284 (1940).

[43] R. W. Hasse and W. D. Myers, Geometrical Relationships of Macroscopic Nuclear Physics (Springer-Verlag, Berlin, 1988).

[44] J. Benlliure et al., Nucl. Phys. A 700, 469 (2002).
[45] J. Benlliure, E. Casarejos, J. Pereira, and K.-H. Schmidt, Phys. Rev. C 74, 014609 (2006).

[46] B. D. Wilkins, E. P. Steinberg, and R. R. Chasman, Phys. Rev. C 14, 1832 (1976).

[47] M. Caamaño, F. Farget, O. Delaune, K.-H. Schmidt, C. Schmitt, L. Audouin, C.-O. Bacri, J. Benlliure, E. Casarejos, X. Derkx, B. Fernandez-Dominguez, L. Gaudefroy, C. Golabek, B. Jurado, A. Lemasson, D. Ramos, C. Rodriguez-Tajes, T. Roger, and A. Shrivastava, Phys. Rev. C 92, 034606 (2015).

[48] G. Chaudhuri and S. Pal, Phys. Rev. C 63, 064603 (2001), and references therein.

[49] G. G. Adamian, N. V. Antonenko, A. Diaz-Torres, and W. Scheid, Nucl. Phys. A 671, 233 (2000).

[50] P. Paul and M. Thoennessen, Ann. Rev. Nucl. Part. Sci. 44, 65 (1994). 